\title{
Analysis of the Influence Factors of Knowledge Transfer in University-Industry Collaborative Innovation
}

\author{
Yanhua Hu \\ Jiangxi University of Finance and Economics, Nanchang, China \\ 15270013762@163.com
}

\begin{abstract}
Keywords: Universities-Industries collaborative innovation; Knowledge transfer; Influence factors; Conceptual model
\end{abstract}

\begin{abstract}
Whether knowledge can be transferred smoothly between the knowledge units determines the performance of collaborative innovation. This paper is aimed at find out the knowledge transfer influence factor in the process of University-Industry collaborative innovation to promote the performance of collaborative innovation. In the situation of University-Industry collaborative innovation, knowledge transfer shows many characteristics which are different from the traditional one. Based on the analysis of the characteristics and process of University-Industry knowledge transfer, this paper put forward a University-Industry knowledge transfer influence factor conceptual model, combined with the perspective of information network and social network. It divided the influence factor into four respects: the factors of knowledge subjects' characteristics, the factors of collaborative innovation network characteristics, the factors of collaborative innovation situation, and the factors of external environment factors in collaborative innovation. Finally, the strategy to promote the knowledge transfer was given based on the analysis results.
\end{abstract}

\section{Introduction}

With the development of economic globalization, technological complexity and variability of innovation increasingly posing challenges to the enterprise Independent innovation. With the development of opening innovation, University-Industry collaborative innovation (hereafter called U-I collaborative innovation), as a new organization of innovative, came into being. U-I collaborative innovation operational mechanism has become a hot issue to many scholars. U-I collaborative innovation aim at achieving value-added knowledge and technological innovation, therefore, knowledge activities throughout the entire process of the collaborative innovation. In the process, the subject of universities and enterprises are continuous interacting and learning to obtain the new knowledge through knowledge transfer. The success of knowledge transfer directly affects the smooth implementation of the U-I collaborative innovation projects. The asymmetric information and intellectual property risks will affect the school-enterprise knowledge transfer initiative, thereby affecting the knowledge transfer effect. Knowledge transfer is a complex system activity; it is susceptible to the surrounding context.

Under the U-I collaborative environment for innovation, knowledge transfer situation has undergone new changes, in order to make U-I collaborative innovation to achieve better performance, it has a theoretical necessity to identify the factors that affect the knowledge transfer performance in U-I collaborative innovation process. Based on knowledge transfer influencing factors analyzed, this paper put forward some strategies to promote knowledge transfer in collaborative innovation.

\section{The Knowledge Transfer in the Process of U-I Collaborative Innovation}

Collaborative innovation was first put forward and given the definition by Peter Glory of MIT Sloan's Center for Collective Intelligence, namely, "the network consisted of self-motivated team with the common vision exchange ideas, information and working conditions with the network cooperate to achieve common goals [1]." Industry-university-research collaborative innovation is that through 
synergy and resource sharing among multiple subjects, results in aggregation effect, accelerates the process of innovation and maximizes the value of innovation [2]. From the perspective of knowledge management, the essence of Industry-university-research collaborative innovation is the change in the way of knowledge production [3]. "Innovative Collaborative Knowledge Production" attracts people with different backgrounds to join the innovation projects by integrating a variety of available resources under Interdisciplinary background. Research collaborative innovation is essentially reflects of the open, dynamic development about Industry-university-research cooperation [4].

U-I collaborative innovation process is accompanied by knowledge transfer. In the process of collaborative innovation, knowledge transfer is no longer a simple linear process, but rather a complex dynamic cycle. Based on previous studies about knowledge transfer process [5, 6], this article put forward a knowledge transfer process model (as shown in Fig. 1).it has three stages: the preparation phase, the operational phase, and the integration and feedback stages. The operational phase is the core of knowledge transfer; it can be divided into knowledge transmission phase, knowledge collaboration stage, knowledge absorption stage.

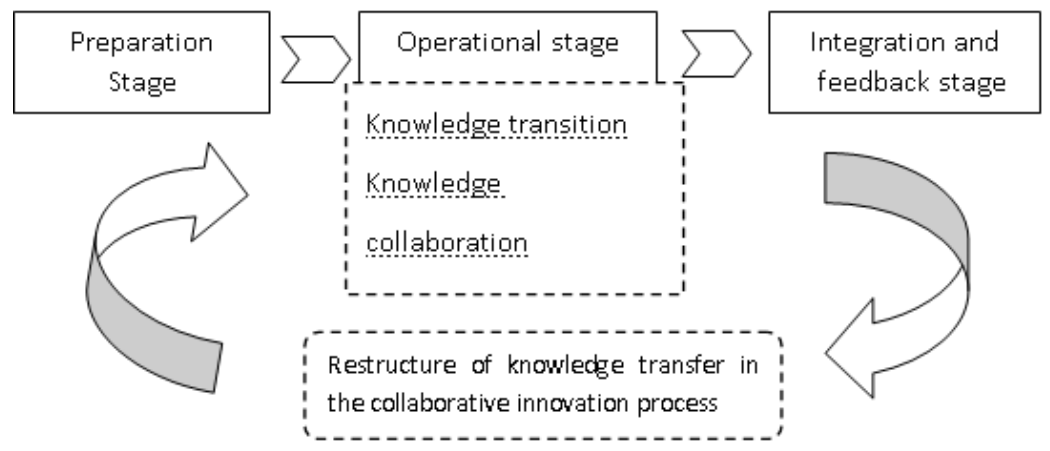

Figure 1. The knowledge transfer process model in U-I collaborative innovation

Preparation stage is the process that enterprises according to their own situation, combined with social and economic environment, decide whether to launch U-I collaborative innovation activities and the choice of partners.

Operational phase is the specific operational stage of knowledge transfer, is the key to generate synergies. At this stage, under the guidance of common goals, universities and industries constantly interacts knowledge, and every interaction can be divided into knowledge transition stage, knowledge collaboration stage, knowledge absorption stage.

Based on the achievement of common goals, U-I collaborative innovation members may terminate the Operational phase. In the integration and feedback stage, members assess the value of knowledge synergy, distribute the benefits, and provide feedback.

\section{Knowledge Transfer Influence Factors in the Process of U-I Collaborative Innovation}

The Conceptual Model. Italian scholar Vito Albino (1999) put forward a four-part framework to analysis knowledge transfer. That is the subjects of knowledge transfer, the situation of knowledge, the content of knowledge transfer and knowledge transfer media [7]. Then, Jeffrey (2003) proposed a simplified analytical framework [8]. Many researchers do some research about the influence factors of knowledge transfer based on above research results. Later, some scholars study this issue from the perspective of social networks.

The knowledge transfer in the process of U-I collaborative innovation is achieved through a series of knowledge interactive activities between knowledge sender (mostly schools) and knowledge of the recipient (mostly enterprises) in certain cooperation environment. According to the analysis of U-I collaborative innovation characteristic, it is obvious that the knowledge transfer will be affected by some new factors Compared with general U-I cooperation. In this paper, Combined with the perspective of information networks and social networks, we proposed the theoretical framework of 
knowledge transfer influence factor model in the process of U-I collaborative innovation(Show in Fig. 2).

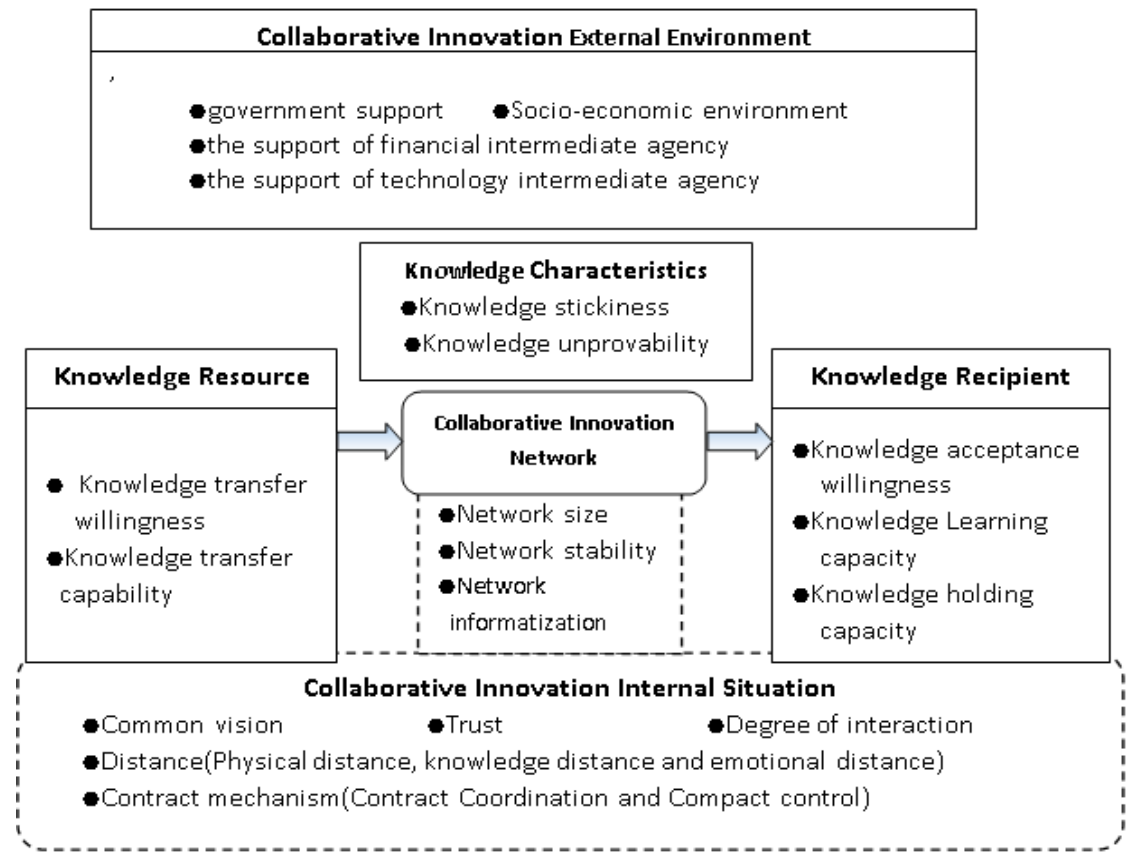

Figure 2. The theoretical framework of knowledge transfer influence factor model in the process of U-I collaborative innovation

Analysis of Influence Factors. This section will analyze the knowledge transfer influence factor in the process of U-I collaborative innovation from four perspective respectively.

The characteristics of collaborative innovation subjects include the characteristics of knowledge, the characteristics of knowledge resource and the characteristics of knowledge recipient.

Knowledge is the object of knowledge transfer in collaborative innovation process. Knowledge Stickiness is mainly composed of tacit knowledge and knowledge complexity, which has a negative influence on knowledge transfer effect. Besides, knowledge usefulness is not knowledge itself, but rather the degree of recognition of the knowledge recipient. The characteristics of knowledge resource include the willingness of knowledge transfer and the knowledge transfer capability. In the process of U-I collaborative innovation, if there is not enough incentive and profit driven, the universities' consciousness of intellectual property right protection will be stronger, knowledge transfer willingness will be weaker, thus affecting the knowledge transfer effect. The knowledge transfer capability of knowledge sender includes knowledge base and the communication ability. If knowledge transfer ability is stronger, the knowledge transfer effect will be better. The characteristics of knowledge recipient include the Willingness of knowledge acceptance, the knowledge learning capacity and the knowledge holding capacity. Knowledge holding capacity reflects the organization's ability to assimilate new knowledge. Only when the new knowledge keeps down enduringly, knowledge transfer is truly effective.

The characteristics of collaborative innovation network include network size, network stability and network information degree.

Network size refers to the amount of school-enterprise collaborative innovation network members. Diverse network system can effectively promote the flow of information to enable members to obtain useful information from more channels. Collaborative innovation network size through affecting the amount of knowledge transferred units indirectly promoting knowledge transfer. Network stability refer to the stable equilibrium consist of the mechanism of resource sharing, risk sharing, complementary advantages, benefit-sharing mutual trust. The stable cooperative relation is conducive to the formation of shared attitudes, beliefs and culture, especially in the process of knowledge transfer, it is easy to form a common technological paradigm. Network information is a measure to 
promote communication. Currie $\mathrm{G}$ pointed out that the use of information technology and IT equipment will effectively promote knowledge sharing and transferring [9]. Mobile Internet and Web 2.0 have a positive impact in usability of knowledge, especially knowledge creation, sharing and transferring ways.

The characteristics of collaborative innovation internal situation include common vision, distance, contract mechanism, trust, degree of interaction.

From the concept of collaborative innovation, a common vision is the basis for collaborative innovation. In the network of U-I collaborative innovation, the clearly expression of common vision is favor of knowledge transfer based on synergies. Distance refers to the degree of closeness between universities and enterprises. It not only refers physical distance, but also it includes knowledge distance, cultural distance, emotional distance between U-I collaborative innovation network members. All these distance play a negetive effect on knowledge transfer. Contract management is an important means of U-I collaborative innovation management. According to management purposes, mainstream research divided contract into two dimensions: contract coordination and contract control. Complete contract coordination mechanism create a good network of explicit knowledge exchange for collaborative innovation members. However, tacit knowledge is difficult to assess, so it is difficult to restrain tacit knowledge formation transfer. Appropriate compact control can play a supervisory role in knowledge transfer; however, strict control contract will reduce knowledge transfer willingness to bring negative effects on knowledge transfer[10]. Effect of trust on knowledge transfer mainly from two aspects: improving knowledge transfer willingness and reduce the difficulty of knowledge transfer. When both sides have a high degree of mutual trust, knowledge source are willing to take certain risks, reduce control and defense to the recipient, and impart useful knowledge more openly; at the same time, the recipient confirm the effectiveness of knowledge provided by knowledge sources due to the reliance on knowledge sources, and are more willing to accept and absorb this knowledge. The communication in the process of knowledge transfer is the exchange process of information or opinions which bear their own knowledge. Communication will accelerate the flow of knowledge, and affect the quantity and quality of the transferred knowledge to enhance the knowledge transfer effect.

The characteristics of collaborative innovation external environment include the degree of government support, the support degree of financial intermediate agency, the support degree of technology intermediate agency and socio-economic environment.

The government formulates relevant policies and regulations and provide economic support to guide and guarantee the smooth implement of U-I collaborative innovation.Financial intermediate agency is a person or organization that plays a media role between supply and demand of funds in the financial markets. Strong financial support is a necessary condition to achieve technological innovation, and financial intermediation is an important way to expand sources of innovative funds. Technology intermediate mainly including productivity promotion centers, science and technology business incubation centers, technology consulting assessment agencies, scientific and technological intelligence and information agencies, which promote effective link among universities, industries and other originations through technical consultation, evaluation and dissemination activities. From the view of economics, the market demand for new technology is the main driving forces for knowledge transfer in collaborative innovation. Advance of knowledge creates new products, and promotes economic growth and the development of the whole society. Conversely, with the driven role of knowledge, social and economic development has generated new knowledge requirements, which play action-oriented role on U-I collaborative innovation, and ultimately affect the result of knowledge transfer.

\section{Conclusion}

This paper describes the knowledge transfer process in U-I collaborative innovation based on system dynamics and put forward a University-Industry knowledge transfer influence factor conceptual 
model. Some strategy to promote knowledge transfer in the process of U-I collaborative innovation was proposed Based on the above analysis.

- Enhance knowledge holding capacity. In the U-I collaborative innovation process, knowledge gained should be use in-depth to improve the effectiveness of knowledge. The improvement of knowledge holding capacity could perpetuate the knowledge transfer results.

- Establish stable collaborative innovation network. In the operation stage of collaborative innovation network, it is important to ensure the stability of the network; stable partnership is conducive to knowledge transfer. In addition, Information degree of the collaborative innovation network to bring more convenient to knowledge interaction.

- Contract and trust combined. In the collaborative innovation alliance with low trust, companies can develop complex, detailed procedures and improve collaboration contract coordination mechanism to gradually increase trust among members, while the partner with high trust could continue to improve coordination function of contract and jointly promote knowledge transfer of the alliance. In addition, enterprises should pay attention to choose the right level of control contract.

\section{Acknowledgements}

This paper was partly supported by the Jiangxi Province Graduate Innovation Special Fund under grant No.YC2015-B052.In addition, I am granted for my tutor for her valuable suggestions. The anonymous reviewers have also considerably contributed to the publication of this paper.

\section{References}

[1] Gloor P, Making the e-Business Transformation, J. Springer Berlin, 2000.

[2] Rao yanting, the connotation, requirements and policy ideas, J. Exploration of Higher Education. 4(2012)29-32.

[3] Dong bobo, Collaborative innovation mode and operation mechanism about universities in China, D. Anhui University, 2014.

[4] Jin huihong, Xue xipeng, Xia Wenlei, Research on operation mechanism of Industry-University - Research collaborative innovation, J. Research on science and technology management. 5(2015)21-25.

[5] Szulanski G, The process of knowledge transfer: A diachronic analysis of stickiness, J. Organizational Behavior and Human Decision Processes, 82 (2000) 9-27.

[6] Gilbert, M., Cordey Hayes, Understanding the process of knowledge transfer to achieve successful technological innovation, J. Tech innovation, 6(1996)301-312.

[7] Albino V., Garavelli A, Schiuma G, Knowledge Transfer and Inter-firm relationships in Industrial Districts: The role of Leader Firm, J. Tech innovation, 19(1998)53-63.

[8] Jeffrey L Cummings, Transferring R\&D knowledge: the key factors affecting knowledge transfer success, J. Journal of Engineering and Technology Management. 20(2003)39-68.

[9] Currie G, Kerrin M, Human resource management and knowledge management: Enhancing knowledge sharing in a pharmaceutical company, J. the International Journal of Human Resource Management, 6(2003)1027-1045.

[10] Diao Lilin, Zhu Guilong. The impact of contract and trust on knowledge transfer in university industry alliances[J]. science research, 2015, 33 (5): 723-733. 